\title{
O ensino de habilidades terapêuticas em FAP: uma revisão de literatura
}

\author{
The teaching of therapeutic skills in FAP: a literature review \\ La enseñanza de habilidades terapéuticas en FAP: una revisión \\ de la literatura
}

\author{
Vinicius Santos Ferreira
}

\begin{abstract}
[1] Universidade Federal de Mato Grosso do Sul - Paranaíba, Pontifícia Universidade Católica de São Paulo (PUC-SP) I Título abreviado: Ensino de habilidades terapêuticas em FAP I Endereço para correspondência: Rua 3, nº 200, Lt.7, Qd. 13, Setor Moraes, CEP 74620-385, Goiânia-GO I Email: viniciusantosferreira@ hotmail.com I doi.org/10.18761/PAC.2020.v11.n2.08
\end{abstract}

Resumo: Nenhuma revisão enfocou exclusivamente em estudos sobre o ensino de FAP para terapeutas. Investigações sobre o ensino de FAP para terapeutas são fundamentais para a sua implementação e disseminação. Este estudo pretendeu realizar uma revisão de literatura com o objetivo de identificar e analisar os estudos empíricos que descreveram, analisaram ou avaliaram estratégias de ensino para a formação de psicólogos em FAP. A busca dos textos ocorreu na plataforma de bases de dados Periódicos CAPES, na base de dados da PEPSIC e no Catálogo de Teses e Dissertações da CAPES, em um site da FAP e nas referências dos estudos. Categorias de análise foram utilizadas para avaliar a qualidade metodológica dos estudos e as estratégias de ensino utilizadas para o treinamento. Foram encontrados 10 artigos e duas dissertações. Foram identificados dois grupos de pesquisa produtivos vinculados a Universidade de Washington e UFPR. Em relação aos aspectos metodológicos, dois estudos foram correlacionais, dois foram estudos de caso e oito empregaram metodologias experimentais. Dos estudos experimentais, quatro utilizaram delineamentos de sujeito único e outros quatro empregaram delineamentos entre grupos. Observou-se a utilização de vários cuidados metodológicos nos dois tipos de estudos experimentais, sendo que o controle experimental aumentou nos estudos mais recentes. As estratégias de ensino utilizadas foram: supervisão (5); treinamento de habilidades em grupo - modalidade online (2) e modalidade presencial (1); e workshop (2).

Palavras-chave: psicoterapia analítica funcional; habilidades terapêuticas; supervisão; treinamento de habilidades; workshop; revisão de literatura. 
Abstract: This paper is a systematic review of the experimental articles published in the Brazilian journal Perspectivas em Análise do Comportamento (RPAC) from 2010, when it was created, to 2018. All experimental research has been considered eligible to be analyzed. The 15 selected investigations revealed: (1) very low number of experimental investigations; (2) human beings as the main kind of subjects; (3) preference for intra-subject design and computerized records; (4) prevalence of basic researches; (5) predominance of research involving stimulus equivalence; (6) limited use of inferential statistics; and (7) little attention to generalization, integrity and agreement between observers in applied research. In addition to this preliminary view on the behavior of research by behavior analysts, the data obtained by the present study suggest the need for greater experimental emphasis.

Keywords: functional analytic psychotherapy; therapeutic skills; supervision; skills training; workshop; literature review.

Resumen: Ninguna revisión se centró exclusivamente en estudios de enseñanza de FAP a terapeutas. Las investigaciones sobre la enseñanza de FAP a los terapeutas son fundamentales para su implementación y difusión. El objetivo de este estudio es realizar una revisión de la literatura para identificar y analizar los estudios empíricos que describieron, analizaron o evaluaron estrategias de enseñanza para la formación de psicólogos en FAP. La búsqueda de los textos tuvo lugar en la plataforma de base de datos periódicos CAPES, en la base de datos PEPSIC y en el Catálogo de Tesis y Disertaciones CAPES, en un sitio web de la FAP y en las referencias de los estudios. Se utilizaron categorías de análisis para evaluar la calidad metodológica de los estudios y las estrategias de enseñanza utilizadas para la capacitación. Encontramos 10 artículos y dos disertaciones. Se identificaron dos grupos de investigación productivos vinculados a la Universidad de Washington y a la UFPR. En cuanto a los aspectos metodológicos, dos estudios fueron correlacionales, dos fueron estudios de casos y ocho emplearon metodologías experimentales. De los estudios experimentales, cuatro usaron diseños de un solo sujeto y otros cuatro emplearon diseños entre sujetos. Se observó el uso de diversas precauciones metodológicas en ambos tipos de estudios experimentales, y el control experimental ha aumentado en estudios más recientes. Las estrategias de enseñanza utilizadas fueron: supervisión (5); entrenamiento de habilidades en grupo: modalidad online (2) y modalidad presencial (1); y workshop (2).

Palabras clave: psicoterapia analítica funcional; habilidades terapéuticas; supervisión entrenamiento de habilidades; workshop; revisión de literatura. 


\section{O ensino de habilidades terapêuticas em FAP: uma revisão de literatura.}

A Psicoterapia Analítica-Funcional (FAP - sigla derivada do inglês) é uma proposta terapêutica originalmente alicerçada nos pressupostos do Behaviorismo Radical (Kohlenberg, Hayes \& Tsai, 1993; Kohlenberg \& Tsai, 1987, 1991), embora atualmente tenha se distanciado de sua formulação inicial (Vandenberghe, 2017). Para Kohlenberg e Tsai (1991), o cerne da mudança terapêutica está na relação terapeuta-cliente. Pressupõe-se que os problemas que ocorrem no cotidiano do cliente também podem ocorrer, via similaridade funcional, na relação terapêutica. O terapeuta usa dessa relação para modelar Comportamentos Clinicamente Relevantes (CCRs) do cliente que ocorrem na sessão, esses são classificados em três tipos: CCRs1 indicam os comportamentos-problema, CCRs2 indicam os comportamentos-melhora e CCRs3, que se referem a comportamentos verbais do cliente de analisar o seu próprio comportamento, estabelecendo relações funcionais entre o responder e os eventos do ambiente. O objetivo desta terapia é modelar CCRs 2 e 3 por reforçamento natural e consequentemente diminuir os CCRs 1.

No primeiro manual para o ensino de FAP, Kohlenberg e Tsai (1991) propuseram que o terapeuta deveria ficar sob controle de cinco regras para atuar como um terapeuta FAP: (a) identificar CRBs; (b) evocar CRBs; (c) reforçar naturalmente aproximações de CRBs 2; (d) verificar se o efeito da intervenção foi reforçador; e (e) interpretar e discutir como as interações em sessão podem se relacionar com as interações fora da sessão. Para seguir essas regras o terapeuta deve desenvolver algumas capacidades específicas além da compreensão teórica do funcionamento da FAP e dos princípios analítico-comportamentais subjacentes, como habilidades terapêuticas relacionadas a intimidade, compaixão, coragem e tomada de risco estratégica (Tsai, Kohlenberg, Kanter, Kohlenberg, Follette \& Callaghan, 2009; Tsai, Yard \& Kohlenberg, 2014).

A integridade de uma proposta psicoterápica, como a FAP, depende da investigação de estratégias de ensino eficazes para uma adequada implemen- tação e disseminação (Southam-Geraw \& McLeod, 2013). A literatura sobre ensino de habilidades terapêuticas é extensa (Herschell, Kolko, Baumann, \& Davis, 2010; Decker, Jameson, \& Naugle, 2011), e diversas estratégias de ensino têm sido investigadas, como: (a) manuais (Miller \& Binder, 2002); (b) supervisão (Alfonsson, Parling, Spannargard, Andersson, \& Lundgren, 2017; Wheeler \& Richards, 2007); (c) treinamento de habilidades de ajuda (Hill \& Lent, 2006); (d) Workshops (Walters, Matson, Baer, \& Ziedonis, 2005); (e) Treinos online (Rousmaniere, 2014); entre outros. Muitos treinamentos que optam por investigar o efeito de pacotes de estratégias de ensino, por exemplo, podem incluir o uso de manual mais supervisão clínica.

Muitas das teorias de ensino-aprendizagem disseminadas na nossa sociedade conceptualizam o aprendiz como um agente passivo, um receptáculo das informações fornecidas por algum especialista, e a finalidade do ensino seria a memorização e a reprodução do conteúdo ensinado (Skinner, 1968). As estratégias de ensino mais comuns, nas quais o aluno tem um papel passivo, seriam o uso de manuais e as aulas expositivas. Outra proposta de ensino é a chamada aprendizagem ativa, em que o estudante se engaja ativamente em atividades de ensino significativas (Prince, 2004). Na aprendizagem ativa o aprender não é apenas receber e recordar informações, o aprendiz é tido como um indivíduo autônomo que aprende ao se engajar em atividades programadas, que exigem interação e reflexão. Segundo Paiva, Parente, Brandão e Queiroz (2016), alguns exemplos de metodologias ativas seriam: grupos de tutoria ou grupos de facilitação, estudo de caso, exercícios em grupo, dramatizações, dinâmicas lúdico pedagógicas, relato crítico de experiência, exposições dialogadas, entre outras.

Muitas dessas metodologias ativas têm sido incorporadas pelas estratégias de ensino que têm sido defendidas teoricamente para o ensino de FAP. Alguns autores defendem que a estratégia de supervisão clínica pode ser ideal para o ensino de FAP (Follette \& Callaghan, 1995; Callaghan, 2006a; Kohlenberg \& Tsai, 1991; Vandenberghe, 2009a). Similarmente ao raciocínio clínico da FAP, que atribuem o foco da mudança à relação terapeuta-cliente, em uma supervisão FAP, a relação supervisor-terapeuta poderia ser utilizada para a mode- 
lagem de repertórios necessários para um terapeuta FAP e para o manejo das dificuldades terapêuticas na condução de casos clínicos particulares. Essa modelagem de comportamentos clinicamente relevantes na própria interação terapeuta-cliente é tida como o principal mecanismo de mudança de uma terapia FAP e funcionaria também como uma estratégia de ensino ativa para o treinamento de terapeutas na relação com o seu supervisor.

Além da supervisão clínica individual, Tsai, Callaghan, Kohlenberg, Follette e Darrow (2009) apresentam dois outros modelos de treinamento em FAP em grupo. Em um primeiro modelo, as supervisões clínicas são realizadas em dois momentos: (1) todo o grupo com o supervisor e (2) em subgrupos, nos quais os próprios alunos se revezam no papel de supervisor e supervisionando. Neste modelo de estratégia de ensino são incluídos procedimentos de ensino ativos como a formulação e a apresentação de caso clínico, incluindo a identificação das próprias dificuldades enquanto terapeuta, com discussão em grupo. Em um segundo modelo de ensino de FAP são adicionadas à estratégia de supervisão clínica outras estratégias de ensino, passivas - como palestras teóricas sobre FAP - e ativas, como: a discussão conceitual do caso clínico do cliente, que seria acessada por gravações de vídeos de sessões clínicas dos próprios clientes ou dos terapeutas; e a utilização de exercícios vivenciais (por exemplo, exercícios para a expressão de sentimentos difíceis ou exercícios de meditação em grupo).

Muitas pesquisas têm sido realizadas para verificar a eficácia da FAP enquanto psicoterapia (ver Kanter et al., 2017; Mangabeira, Kanter \& Del Prette, 2012; Tsai, Yard, \& Kohlenberg, 2014), porém menos esforços têm sido dedicados para o desenvolvimento de estratégias de ensino eficazes dessa proposta psicoterapêutica. Em uma revisão de literatura sobre estudos empíricos em FAP, Kanter et al. (2017) encontraram apenas 3 estudos sobre o ensino dessa proposta psicoterápica, embora os critérios de inclusão não tenham abarcado importantes periódicos brasileiros e estudos com menor rigor metodológico, o que pode ter subestimado a produção real na área.

Este estudo objetivou, por meio de uma revisão de literatura, identificar e analisar estudos empíricos que descreveram, analisaram ou avaliaram es- tratégias de ensino para a formação de psicólogos em Psicoterapia Analítica Funcional (FAP). Dois aspectos serão analisados, a qualidade metodológica dos estudos e as estratégias de ensino utilizadas para o ensino de FAP.

\section{Método}

\section{Fonte}

As fontes utilizadas neste trabalho foram manuscritos científicos em formatos de artigos, teses ou dissertações.

\section{Estratégias de Busca}

A estratégia de busca envolveu quatro etapas: (a) busca em plataformas e bases de dados de periódicos científicos; (b) busca em uma base de teses e dissertações; (c) busca em site com lista de publicações da FAP; e (d) busca nas referências bibliográficas dos manuscritos encontrados. O período de busca abrangeu todas as publicações até o final de 2018.

Plataformas de busca em bases de dados. Uma busca sistemática foi realizada na plataforma de bases de dados Periódicos CAPES e na base de dados da PEPSIC. A plataforma Periódicos CAPES foi selecionada por incluir bases de dados relevantes da literatura nacional e internacional. A opção pela base de dados da PEPSIC foi pelo seu acervo com periódicos nacionais e latino-americanos importantes sobre Análise do Comportamento, como: a Revista Brasileira de Terapia Cognitiva e Comportamental, a Acta Comportamentalia e a Revista Perspectivas em Análise do Comportamento. A busca no portal do Periódico CAPES ocorreu na busca avançada por assunto, em qualquer local do texto, com os termos exatos e utilizando o algoritmo de busca: therap* training AND "Functional Analytic Psychotherapy". Essa busca ocorreu no dia 02/06/2018. A busca na base de dados da PEPSIC ocorreu na pesquisa por artigos em todos os índices com o termo único "FAP". Essa busca ocorreu no dia 06/06/2018.

Base de teses e dissertações. Com o intuito de incluir pesquisas de qualidade ainda não publicadas 
em periódicos foram inclusas teses e dissertações. Foi escolhido o Catálogo de Teses e Dissertações da CAPES que contém um grande volume de teses e dissertações publicadas no Brasil. O Currículo Lattes dos autores das teses e dissertações encontradas foi inspecionado para verificar se estas já haviam sido publicadas, em caso positivo o artigo seria incluído e a tese ou dissertação não.

Site da FAP. O site https://functionalanalyticpsychotherapy.com/references/ mantém uma listagem das publicações atualizadas em FAP identificadas pelo grupo de autores responsáveis pela site.

Referências. Os manuscritos encontrados com as três estratégias anteriores tiveram as suas referências inspecionadas em busca de novos artigos. Parte-se do pressuposto que um artigo sobre ensino de FAP, revise outros artigos com objetivo semelhante e os descreva, principalmente, na introdução e na discussão. Caso um novo artigo fosse encontrado, suas referências seriam, por sua vez, examinadas, assim sucessivamente. Essa estratégia tem sido denominada de método de bola de neve "snowball method" (Greenhalgh, Robert, MacFarlane, Bate, \& Kyriakidou, 2004) devido ao seu caráter cumulativo, que toma vantagem do conhecimento e da revisão de outros autores sobre o mesmo tema. O método de busca nas referências seguiu dois procedimentos: (a) durante a leitura integral do corpo do texto, foram inspecionadas, de forma assistemática, citações de outros estudos que indicassem que estes poderiam se tratar de ensino de FAP; (b) as palavras chave de busca usadas anteriormente foram buscadas nos títulos dos textos nas referências bibliográficas.

\section{Critérios de Inclusão e Exclusão}

Após a leitura cuidadosa dos textos selecionados, foram adotados os seguintes critérios de inclusão: (1) deveriam ser estudos empíricos; (2) com ao menos um dos seguintes objetivos - (a) descrever (e.g., estudo de caso), (b) analisar (e.g., estudos correlacionais) ou (c) avaliar por meio de manipulações experimentais (e.g., estudos experimentais) estratégias de ensino para a formação de terapeutas em FAP. Os seguintes critérios de exclusão foram definidos: (a) estudos que não trataram do ensino de habilidades terapêuticas; (b) estudos teóricos (ex.: conceituais, históricos), revisões de literatura e meta-análises; (c) estudos que não analisaram modelos de terapia baseados na FAP; (d) estudos que treinaram populações ou profissionais que não sejam estudantes de psicologia ou psicólogos; e (e) textos que foram escritos em outro idioma que não inglês ou português.

\section{Procedimento de Análise}

Todos os manuscritos selecionados foram lidos na íntegra e as informações foram analisadas segundo um sistema de variáveis e categorias desenvolvido especificamente para os propósitos desse estudo. As variáveis analisadas foram definidas antes da leitura dos estudos, considerando os aspectos de interesse desta revisão, que compuseram três eixos de análise: características bibliográficas, aspectos metodológicos e características das estratégias de ensino. Cada eixo agrupou um conjunto de variáveis de interesse. As variáveis foram analisadas segundo categorias de análise ou, em alguns casos, com a transposição literal da informação do texto analisado (ex.: nome da revista). As categorias de análise foram definidas após a leitura dos textos analisados. As informações relativas a cada variável foram anotadas para cada estudo, e, posteriormente, elas foram agrupadas, segundo uma análise qualitativa, em categorias que fossem significativas para estabelecer semelhanças ou diferenças entre os estudos. Todas as informações registradas relativas a cada variável foram exaustivamente alocadas nas categorias apresentadas, o que significa que informações que não enquadrem nessas categorias não tiveram ocorrência em nenhum estudo revisado. A Tabela 1 descreve as variáveis contidas em cada eixo de análise e suas respectivas formas de registro ou categorias de análise. 
Tabela 1. Variáveis analisadas e suas formas de registro ou categorias de análise por eixo.

\begin{tabular}{|c|c|c|}
\hline Eixos & Variáveis & Formas de registro/categorias de análise \\
\hline \multirow{7}{*}{$\begin{array}{l}\text { Características } \\
\text { bibliográficas }\end{array}$} & Ano de publicação & Registro conforme artigo. \\
\hline & Autoria & Registro conforme artigo. \\
\hline & Filiação institucional dos autores & Registro conforme artigo. \\
\hline & Origem (país) das instituições & Registro conforme artigo. \\
\hline & Idioma da redação do manuscrito & Categorias: (a) inglês; (b) português. \\
\hline & $\begin{array}{l}\text { Veículo de publicação/instituição } \\
\text { (no caso de dissertações) }\end{array}$ & Registro conforme artigo. \\
\hline & Citações entre manuscritos & $\begin{array}{l}\text { Registro do } n^{\circ} \text { de manuscritos incluídos nesta revisão que citaram outros } \\
\text { manuscritos incluídos nesta revisão. }\end{array}$ \\
\hline \multirow{8}{*}{$\begin{array}{l}\text { Características } \\
\text { das estratégias } \\
\text { de ensino }\end{array}$} & Tipo de estratégias de ensino ${ }^{a}$ & $\begin{array}{l}\text { Categorias: (a) supervisão; (b) workshop - curso intensivo de curta duração } \\
\text { com atividades práticas; (c) treinamento de habilidades terapêuticas [THT] } \\
\text { - treino pré-prático, entre o ensino conceitual e a prática, que objetiva } \\
\text { o desenvolvimento de habilidades de ajuda ou terapêuticas [Hill \& Lent, } \\
\text { 2006], ele se distingue do workshop por não ser intensivo e prover acompa- } \\
\text { nhamento do aluno. }\end{array}$ \\
\hline & Modalidade do treino & Categorias: (a) presencial; (b) online por videoconferência. \\
\hline & $\begin{array}{l}\text { Procedimentos de ensino utiliza- } \\
\text { dos pelo supervisor / facilitador }\end{array}$ & $\begin{array}{l}\text { Categorias: (a) manejo da relação com o terapeuta - modelagem; (b) exer- } \\
\text { cícios vivenciais - ensaio comportamental, role play e exercícios com situa- } \\
\text { ções análogas; (c) psicoeducação - apresentações teóricas; (d) discussão } \\
\text { de dificuldades do terapeuta com uso de feedback e/ou modelação; (e) } \\
\text { leituras didáticas; (f) tarefa de casa; (g) exemplo de caso clínico; (h) treino } \\
\text { em mindfulness; e (i) reestruturação cognitiva. }\end{array}$ \\
\hline & Uso de protocolo & Categorias: (a) sim; (b) não. \\
\hline & $N^{\circ}$ de facilitadores por treino & Registro conforme artigo. \\
\hline & $\mathrm{N}^{\circ}$ de participantes por treino & Registro conforme artigo. \\
\hline & Duração total do treino & Registro conforme artigo. \\
\hline & Número de sessões de treino & Registro conforme artigo. \\
\hline \multirow{6}{*}{$\begin{array}{l}\text { Aspectos } \\
\text { metodológicos }\end{array}$} & Tipo de método & $\begin{array}{l}\text { Categorias: (a) estudo de caso - descrição da intervenção com análise } \\
\text { qualitativa; (b) método correlacional - análise da variação conjunta de variá- } \\
\text { veis sem manipulação experimental; (c) método experimental - manipulação } \\
\text { experimental e análise quantitativa dos dados. }\end{array}$ \\
\hline & $\begin{array}{l}\text { Delineamento experimental } \\
\text { (apenas para os estudos que } \\
\text { utilizaram método experimental) }\end{array}$ & $\begin{array}{l}\text { Categorias e subcategorias: (1) delineamento de sujeito único: (a) AB; (b) } \\
\text { de reversão - ABA ou variações; (c) de linha de base múltipla. (2) deline- } \\
\text { amento entre grupos: (a) pré-experimental - apenas uma condição com } \\
\text { avaliação pré e pós-teste; e (b) experimental - ensaio clínico randomizado. }\end{array}$ \\
\hline & Follow up & $\begin{array}{l}\text { Categorias: (a) Não; (b) Sim, nesse caso o período do follow up foi descri- } \\
\text { to. }\end{array}$ \\
\hline & $\mathrm{N}^{\circ}$ de treinandos total (amostra) & Registro conforme artigo. \\
\hline & Habilidades medidas (VD) ${ }^{\mathrm{b}}$ & $\begin{array}{l}\text { Categorias: (a) pacote multicomponente de manejo de comportamento } \\
\text { clinicamente relevantes (chamado nesse estudo de habilidades FAP) - incluí } \\
\text { identificar, evocar e modelar CCRs; (b) habilidades isoladas da FAP - identi- } \\
\text { ficar, evocar ou modelar CCRs, especificar qual; (c) intimidade; (d) empatia; } \\
\text { (e) mindfulness-traço; (f) amor; e (g) autenticidade. }\end{array}$ \\
\hline & $\begin{array}{l}\text { Procedimentos e instrumentos } \\
\text { utilizados para medir a VD }\end{array}$ & $\begin{array}{l}\text { Categorias: (a) instrumento de autorrelato; (b) avaliação por exercícios; (c) } \\
\text { avaliação teórica; e (d) observação direta de comportamentos, especificar } \\
\text { se: (1) na sessão ou em cenário de role play; (2) em vídeo ou sala de espe- } \\
\text { lho; (3) com registro por checklist, sistemas de categorizações ou escalas. }\end{array}$ \\
\hline
\end{tabular}

Notas. ${ }^{a}$ A variável procedimento de ensino se diferencia da variável estratégia de ensino por especificar os componentes, em termos de procedimentos, daquela estratégia de ensino particular. ${ }^{\mathrm{b}} \mathrm{A}$ variável habilidades medidas contempla quais são os construtos ou comportamentos que estão sendo medido como variável dependente (VD); a variável procedimentos e instrumentos utilizados para medir a VD responde a questão "como" a VD está sendo medida. 


\section{Resultados e Discussão}

A Figura 1 apresenta a diagramação do fluxo de artigos encontrados no processo de seleção dos manuscritos, todas as estratégias de busca foram inclusas aqui.
O fato de que nenhum novo artigo ter sido encontrado pelo método de busca pelas referências foi tratado como um indicativo de que os outros métodos de busca foram adequados, visto que contemplaram todos os artigos citados pelos estudiosos da temática e ainda adicionaram outros artigos.

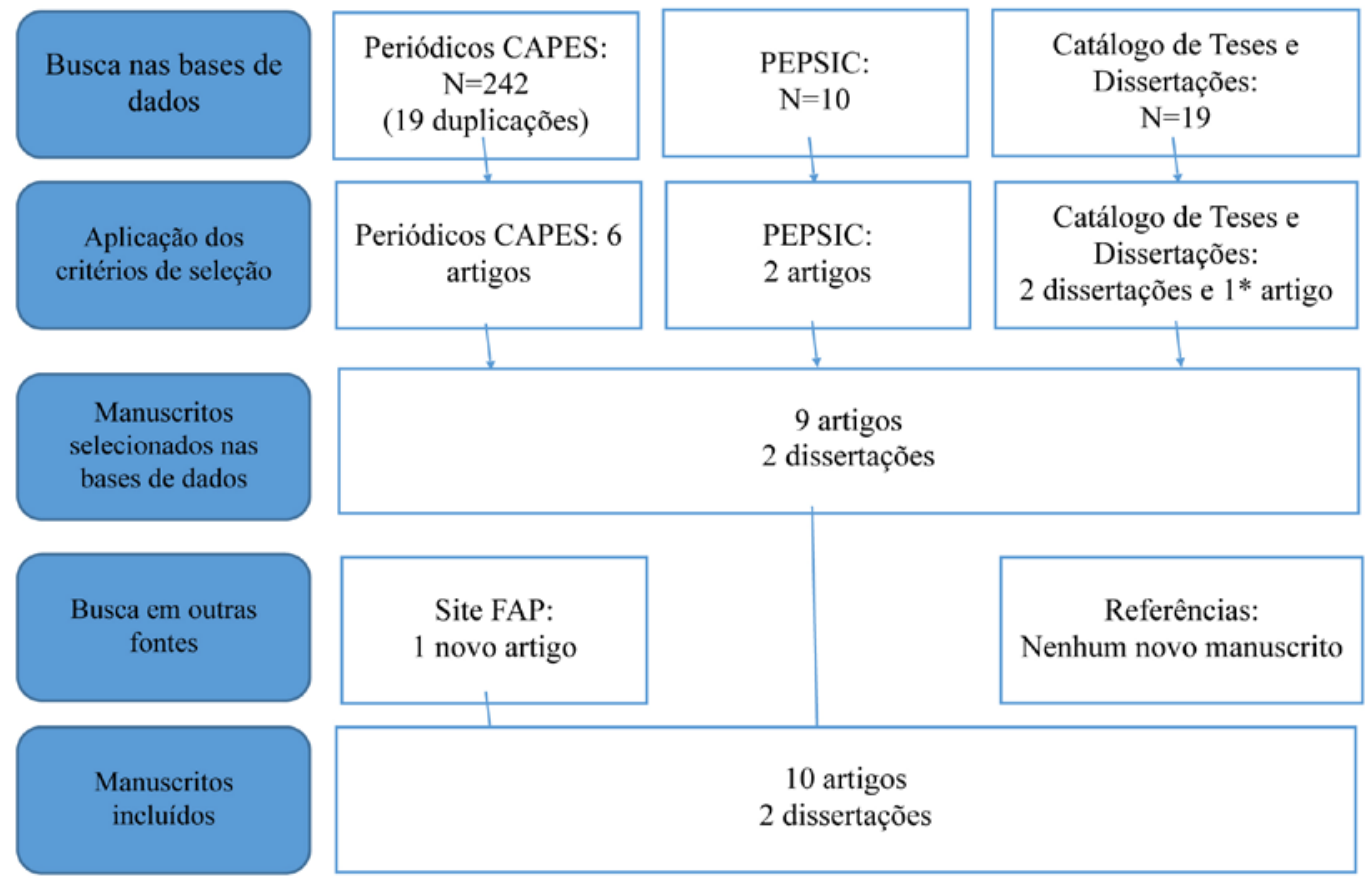

Figura 1. Diagrama de fluxo de distribuição de artigos encontrados antes e após refinamento. *Uma das dissertações encontradas foi publicada em formato de artigo, optou-se por manter o artigo.

*Uma das dissertações encontradas foi publicada em formato de artigo, optou-se por manter o artigo.

\section{Características Bibliográficas.}

Foram encontrados manuscritos de pesquisadores de instituições do Brasil $(\mathrm{n}=7)$, dos Estados Unidos $(n=6)$ e de Singapura $(n=1)$. Houve sobreposição no número de manuscritos por países devido a duas colaborações internacionais, uma entre instituições do Brasil e dos Estados Unidos (Silveira, et al., 2009) e outra entre instituições dos Estados Unidos e de Singapura (Keng, et al., 2016). O idioma de publicação dos manuscritos foi o português para seis dos artigos e o inglês para os outros seis trabalhos. Dos 10 artigos, três foram publicados no periódico International Journal of Behavioral Consultation and Therapy, dois foram publicados na Revista Brasileira de Terapia Cognitivo e Comportamental e os outros artigos foram publicados em diferentes periódicos - Perspectivas em Análise do Comportamento, Psychotherapy, Clinical Psychology and Psychotherapy, Acta Comportamentalia e Psychological Record. As duas dissertações foram realizadas na Universidade Federal do Paraná (UFPR) e na Universidade de São Paulo (USP).

Ao analisar esses dados em conjunto, parece haver uma divisão quase igualitária entre a produção de pesquisas sobre treinamento de psicoterapeutas em FAP por pesquisadores de instituições do Brasil e dos Estados Unidos e em relação as publicações em inglês e português. As colaborações internacionais foram raras e parece ter havido pouco intercâmbio de publicações dos pesquisadores em periódicos estrangeiros, com exceção de Silveira et al. (2009) e Wielenska e Oshiro (2012). Isso indica 
que o idioma ainda pode ser um entrave no acesso a produção na área. Uma análise das citações dos manuscritos de pesquisadores de instituições brasileiras e estadunidenses indicou que os manuscritos produzidos nos Estados Unidos não citaram nenhum estudo produzido no Brasil, ao passo que os estudos brasileiros citaram os estadunidenses 7 vezes (para essa análise foi excluído o artigo de colaboração entre instituições dos dois países). É provável que os pesquisadores de instituições estadunidenses tenham pouco acesso aos estudos brasileiros nessa área. É possível que essas interpretações possam ser enviesadas pelos critérios de busca que incluíram apenas estudos em português ou inglês.

Observou-se que alguns pesquisadores participaram da autoria de mais de um manuscrito. Jonathan Kanter da Universidade de Washington e Jocelaine Silveira da UFPR participaram da autoria de três manuscritos cada, sendo que a pesquisadora brasileira também foi orientadora da dissertação de Meurer (2011). Mavis Tsai e Robert Kohlenberg, também da Universidade de Washington, participaram da autoria de dois artigos cada, todos os outros pesquisadores foram autores apenas de um manuscrito. Outros cinco autores, além dos nomes citados, também são vinculados a Universidade de Washington ou ao Evidence-Based Practice Institute, ambos em Seattle. Essas duas instituições também foram vinculadas a quatro dos 12 manuscritos publicados, ao passo que, em território brasileiro, se destacam a UFPR também com quatro publicações e a USP com duas. Outras instituições foram vinculadas a apenas uma publicação. Esse cenário pode indicar alguns grupos de pesquisa que têm se dedicado a essa temática. Aparentemente, esses grupos de pesquisa parecem estar concentrados em polos fixos, principalmente Seattle e Curitiba, e não desenvolver projetos entre si e esporadicamente com pesquisadores de outras localidades.

Por fim, foi feita uma análise da data de publicação dos manuscritos. O primeiro estudo empírico publicado sobre treinamento de terapeutas em FAP foi publicado no Brasil, por Sousa e Vandenberghe, em 2007, vinte anos após a primeira publicação da FAP (Kohlenberg \& Tsai, 1987) e muitos anos depois das primeiras publicações conceituais sobre o treinamento de terapeutas em FAP, que ocorreram com o lançamento do primeiro manual da FAP
(Kohlenberg \& Tsai, 1991) e o artigo de Follette e Callaghan (1995) sobre supervisão terapêutica. Após essa primeira publicação as outras se seguiram por mais 10 anos, até 2017, com ausências de publicações nos anos de 2008, 2010 e 2014 e com mais de uma publicação apenas nos anos de $2012 \mathrm{e}$ 2016, com, respectivamente, dois e quatro manuscritos publicados, o que parece indicar um aumento nos estudos da área após os 5 primeiros anos (com apenas 3 publicações, todas de instituições brasileiras, uma delas em colaboração).

\section{Características das Estratégias de Ensino} $\mathrm{Na}$ Tabela 2 foram descritas características das estratégias de ensino utilizadas pelos estudos selecionados para o treinamento em FAP. Dois estudos (Knott et al., 2015; Terry \& Kohlenberg, 2012) não foram incluídos nessa tabela por utilizar uma amostra que passou por diferentes formas de treino em FAP e por não oferecer uma descrição adequada das estratégias de ensino.

Os estudos analisados na Tabela 2 avaliaram três estratégias de ensino diferentes: cinco estudos de supervisão, três estudos de treinamento de habilidades terapêuticas (THT) - também conhecido na literatura como treinamento de habilidades de ajuda (helping skills training, Hill \& Lent, 2006) - e dois estudos de workshop. Em oito estudos, as estratégias de ensino foram presenciais e em dois deles a modalidade de treino foi online (os dois foram treinamentos de habilidades terapêuticas) usando tecnologia de conferência pela internet. A investigação de determinadas estratégias de ensino parece se relacionar com determinadas instituições e grupos de pesquisa, os três estudos de THT são americanos, enquanto todos os estudos de workshop e supervisão são brasileiros (com uma colaboração internacional - Silveira et al., 2009).

Em relação a diversidade de tipos de treino, houve uma preferência pela investigação de estratégias de ensino ativas, prático-vivenciais, como supervisões, THTs e workshops, que tem se mostrado mais efetivas (Beidas \& Kendall, 2010; Beidas, Koerner, Weingardt, \& Kendall, 2011), e que são preferidas pelos autores da FAP (Tsai, Yard, \& Kohlenberg, 2014). Nenhum estudo investigou o efeito de manuais ou comparou as estratégias vivenciais com o uso de manuais. Embora o uso de 
manuais seja a estratégia mais comumente utilizada para treinar psicoterapeutas, a carência de estudos que investiguem essa estratégia já é bem documentada por outras revisões (Herschell et al., 2010; Miller \& Binder, 2002). Também chama atenção a falta de estudos que utilizam pacotes de estratégias de ensino multicomponentes, que combinam diferentes estratégias, uma vez que essas são as estratégias mais comuns na literatura e com os melhores resultados (Herschell et al., 2010). O uso de estratégias de ensino baseadas em computadores para o ensino de FAP tem sido apenas parcialmente explorado. Berger (2004) propõe duas formas de se utilizar computadores para o ensino de psicoterapia para: (a) mediar a comunicação entre treinadores e treinandos; e (b) para entregar informações que facilitem o estudo individual - como manuais, videoaulas, sessão de terapias por vídeo, ou ainda ambientes de aprendizagem interativos, como o atual Coursera. Apenas a primeira forma foi explorada nos estudos de FAP.

Tabela 2. Caracterização das estratégias de ensino por estudo analisado.

\begin{tabular}{|c|c|c|c|c|c|c|c|c|c|c|c|c|}
\hline \multirow[t]{2}{*}{ Manuscrito } & \multirow{2}{*}{$\begin{array}{l}\text { Estratégia } \\
\text { de ensino - } \\
\text { Modalidade } \\
\text { do treino }\end{array}$} & \multirow{2}{*}{$\begin{array}{l}\mathrm{N}^{\circ} \text { de } \\
\text { particip. } \\
\text { por } \\
\text { treino }\end{array}$} & \multirow{2}{*}{$\begin{array}{l}N^{\circ} \text { de } \\
\text { facilitadores } \\
\text { por treino }\end{array}$} & \multirow{2}{*}{$\begin{array}{l}\text { Duração } \\
\text { total do } \\
\text { treino }\end{array}$} & \multirow{2}{*}{$\begin{array}{l}\mathrm{N}^{\circ} \text { de } \\
\text { sessões }\end{array}$} & \multirow{2}{*}{$\begin{array}{l}\text { Uso de } \\
\text { protocolo }\end{array}$} & \multicolumn{6}{|c|}{ Procedimento de ensino } \\
\hline & & & & & & & $\begin{array}{l}\text { Manejo } \\
\text { da } \\
\text { relação }\end{array}$ & $\begin{array}{l}\text { Exercícios } \\
\text { vivenciais }\end{array}$ & $\begin{array}{l}\text { Psico } \\
\text { educação }\end{array}$ & $\begin{array}{l}\text { Discussão } \\
\text { de dificul- } \\
\text { dades }\end{array}$ & $\begin{array}{l}\text { Leitura } \\
\text { didática }\end{array}$ & Outros \\
\hline $\begin{array}{l}\text { Kanter et al. } \\
(2013)\end{array}$ & THT - online & 4 & 2 & 16 horas & 8 & Sim & Sim & Sim & Sim & Sim & Não & $\begin{array}{l}\text { Tarefa de casa; } \\
\text { exemplo de caso } \\
\text { clínico; e treino } \\
\text { em mindfulness. }\end{array}$ \\
\hline $\begin{array}{l}\text { Keng et al. } \\
(2016)\end{array}$ & $\begin{array}{l}\text { THT - pre- } \\
\text { sencial }\end{array}$ & $6-7$ & 2 & 12 horas & 8 & Sim & Sim & Sim & Sim & Sim & Sim & $\begin{array}{l}\text { Tarefa de casa; } \\
\text { exemplo de caso } \\
\text { clínico; e treino } \\
\text { em mindfulness. }\end{array}$ \\
\hline $\begin{array}{l}\text { Maitland et al. } \\
\text { (2016) }\end{array}$ & THT - online & 8 & 2 & 16 horas & 8 & Sim & Sim & Sim & Sim & Sim & Não & $\begin{array}{l}\text { Tarefa de casa; } \\
\text { exemplo de caso } \\
\text { clínico; e treino } \\
\text { em mindfulness. }\end{array}$ \\
\hline $\begin{array}{l}\text { Almeida et al. } \\
\text { (2016) }\end{array}$ & $\begin{array}{l}\text { Workshop - } \\
\text { presencial }\end{array}$ & 6 & 1 & 5 horas & 1 & Sim & Não & Sim & Sim & Não & Não & $\begin{array}{l}\text { Exemplo de } \\
\text { caso clínico. }\end{array}$ \\
\hline $\begin{array}{l}\text { Fonseca } \\
\text { (2016) }\end{array}$ & $\begin{array}{l}\text { Workshop - } \\
\text { presencial }\end{array}$ & 23 & 1 & 16 horas & 2 & Sim & Não & Sim & Sim & Sim & Sim & Não \\
\hline $\begin{array}{l}\text { Silveira et al. } \\
(2009)\end{array}$ & $\begin{array}{l}\text { Supervisão - } \\
\text { presencial }\end{array}$ & 1 & 1 & $\begin{array}{l}8 \text { horas } \\
\text { e } 20 \\
\text { minutos }\end{array}$ & 10 & Não & Sim & Não & Sim & Sim & Sim & Não \\
\hline $\begin{array}{l}\text { Meurer } \\
\text { (2011) }\end{array}$ & $\begin{array}{l}\text { Supervisão - } \\
\text { presencial }\end{array}$ & 1 & 1 & 4 horas & 4 & Sim & Não & Não & Sim & Sim & Não & Não \\
\hline $\begin{array}{l}\text { Lepienski e } \\
\text { Silveira(2017) }\end{array}$ & $\begin{array}{l}\text { Supervisão - } \\
\text { presencial }\end{array}$ & 1 & 1 & - & 8 & Não & Sim & Não & Não & Sim & Não & Não \\
\hline $\begin{array}{l}\text { Sousa \& } \\
\text { Vandenberghe } \\
\text { (2007) }\end{array}$ & $\begin{array}{l}\text { Supervisão - } \\
\text { presencial }\end{array}$ & 1 & 1 & - & - & Não & Sim & Não & Não & Sim & Não & $\begin{array}{l}\text { Questionamento } \\
\text { socrático. }\end{array}$ \\
\hline $\begin{array}{l}\text { Wielenska \& } \\
\text { Oshiro (2012) }\end{array}$ & $\begin{array}{l}\text { Supervisão - } \\
\text { presencial }\end{array}$ & 14 & 1 & - & - & Não & Sim & Não & Sim & Sim & Não & Não \\
\hline
\end{tabular}

Dos cinco estudos de supervisão, apenas um (Meurer, 2011) apresentou um protocolo com os procedimentos de ensino descritos detalhadamente por encontro, os outros descreveram os procedimentos de maneira genérica e sem uma ordem clara (Bootzin \& Ruggill, 1988; Hill \& Lent, 2006). Os procedimentos mais utilizados foram a discussão de dificuldades do terapeuta com uso de feedback ou modelação (cin- co estudos) e o manejo da relação com o terapeuta (4 estudos); nenhum desses estudos utilizou exercícios vivenciais, três estudos utilizaram psicoeducação e apenas um utilizou leituras didáticas.

Ao contrário dos estudos de supervisão, todos os estudos de THT e de workshop apresentaram descrições protocoladas e utilizaram exercícios vivenciais, psicoeducação e exemplos de casos clí- 
nicos como procedimentos de ensino. Isso indica uma diferença importante entre esses métodos de ensino. Os THTs e os workshops optaram pelo uso de: procedimentos de ensino em contextos simulados ou análogos ao contexto clínico - método evitado pela estratégia de supervisão - e métodos de exposição oral teórica, utilizando a psicoeducação, com exemplos de caso clínico, mais frequentemente que os estudos de supervisão.

Os três estudos de THT foram muito semelhantes, pois envolveram a investigação do mesmo protocolo de intervenção (Kanter et al., 2012), utilizando diversas estratégias de ensino, com o foco em estratégias de ensino ativas, como prática deliberada, feedback, modelação ao vivo por reforçamento natural e tarefas de casa - para o desenvolvimento de habilidades terapêuticas interpessoais, ao invés de um treino conceitual e teórico (Beidas \& Kendall, 2010; Kanter et al., 2012). Esses estudos de THT se diferenciam dos estudos de workshops por utilizar procedimentos de manejo de relação e tarefas de casa, que são possibilitadas pelo formato do treino distribuído ao longo de semanas, o que permite um acompanhamento das particularidades dos terapeutas. O ensino de mindfulness ocorreu apenas nos estudos americanos de THT, o que pode mostrar uma não aderência da comunidade brasileira as estratégias de mindfulness (Vandenberghe, 2011), apesar de ser uma habilidade indicada para o terapeuta FAP (Tsai, et al. 2009).

Ainda em relação aos procedimentos de ensino, duas questões chamam a atenção. Primeiro, todos os estudos investigaram a utilização de múltiplos procedimentos de ensino ao invés de investigar o efeito de procedimentos de ensino isoladas; essa combinação de efeitos parece produzir os melhores resultados (Hill \& Lent, 2006). Segundo, em sete dos 10 estudos utilizou-se o manejo da relação por modelagem dos comportamentos relevantes que o terapeuta emite na relação com o supervisor, no caso de supervisão, ou com o facilitador (ou mesmo colegas de treino, ver Vandenberghe, 2009b), no caso de treinos em grupo. Esse procedimento de ensino simula o funcionamento de uma terapia FAP e é recomendado por vários autores (Callaghan, 2006; Follette \& Callaghan, 1995; Tsai et al., 2009; Tsai, Yard \& Kohlenberg, 2014) para a formação de um terapeuta em FAP.
Em relação à estrutura dos treinamentos, houve uma uniformidade maior nos estudos de THT, devido a utilização do mesmo protocolo, e uma maior variação nos estudos de workshop e supervisão. Os estudos de THT contaram com dois treinadores e grupos pequenos (características do treino recomendadas por Hill \& Lent, 2006), com variação de quatro a oito participantes por treino; todos tiveram oito sessões com duração total de 12 a 16 horas de treino. Os dois workshops foram ministrados por apenas um facilitador e contaram com grupos de seis e 23 participantes - esse tamanho de grupo pode dificultar uma adequada condução do grupo e uma atenção individualizada; um dos treinos durou 16 horas e foi dividido em dois encontros e o outro durou apenas 5 horas em um único encontro. Todos os estudos de supervisão contaram com um supervisor e quatro deles foram feitos individualmente, no estudo de Wielenska e Oshiro (2012) o supervisor atendia 14 alunos simultaneamente; dos estudos de supervisão que relataram a duração e o número de sessões, o número de sessões variou de quatro a 10 e as horas de supervisão de 4 a 8 e 20 minutos de duração.

A duração de todos os treinos foi relativamente pequena, variando de 4 a 16 horas. As habilidades a serem desenvolvidas para a capacitação de um terapeuta em um modelo psicoterápico são complexas, o que talvez exija a investigação de treinos de maior duração e a investigação de combinações de estratégias de ensino, ou ainda, de programas de treinamento dessas habilidades de longa duração, como pós-graduações.

\section{Aspectos Metodológicos}

Para essa análise, os estudos foram divididos conforme o tipo de metodologia empregada, visto que cada tipo de método tem limitações e objetivos particulares. Dos manuscritos incluídos: oito utilizaram métodos experimentais, dois descreveram estudos de caso e dois utilizaram métodos correlacionais.

Estudos experimentais. A Tabela 3 descreve os aspectos metodológicas analisados nos estudos experimentais. 
Tabela 3. Aspectos metodológicas dos estudos analisado.

\begin{tabular}{|c|c|c|c|}
\hline Manuscrito & $\begin{array}{l}\text { Habilidades } \\
\text { medidas (VD) }\end{array}$ & $\begin{array}{l}\text { Tipos de Instrumentos utiliza- } \\
\text { dos para medir a VD } \\
\text { Tipo Grupos Amostra Teste } 1 \\
\text { Treino Teste } 2 \text { Treino Teste } 3\end{array}$ & Delineamentos entre grupos \\
\hline $\begin{array}{l}\text { Kanter et al. } \\
(2013)\end{array}$ & $\begin{array}{l}\text { Habilidades } \\
\text { FAP }\end{array}$ & $\begin{array}{l}\text { Autorrelato (FAPIS) e exercício } \\
\text { de caso clínico (FAP-Vinhetas }{ }^{a} \text { ) }\end{array}$ & $\begin{array}{l}\text { Ensaio clínico randomizado/ GE }(n=8) \text { O X O } \\
\text { controle com lista de espera GC-LE }(n=8) \text { O O X O }\end{array}$ \\
\hline $\begin{array}{l}\text { Keng et al. } \\
(2016)\end{array}$ & $\begin{array}{l}\text { Habilidades } \\
\text { FAP, empatia, } \\
\text { mindfulness- } \\
\text {-traço, amor e } \\
\text { autenticidade }\end{array}$ & $\begin{array}{l}\text { Autorrelato (FAPIS, IRI, CLS, } \\
\text { FFMQ-SF, AS, TEI e MC-SDS), } \\
\text { e exercício de caso clínico (FAP- } \\
\text { Vinhetas }^{\mathrm{a}} \text { ) }\end{array}$ & $\begin{array}{l}\text { Ensaio clínico randomizado/ GE }(n=13) \text { O X O O (follow up) } \\
\text { controle com lista de espera GC-LE }(n=12) \text { O O O (follow up) } \\
2 \text { meses }\end{array}$ \\
\hline $\begin{array}{l}\text { Maitland et al. } \\
\text { (2016) }\end{array}$ & $\begin{array}{l}\text { Habilidades } \\
\text { FAP }\end{array}$ & $\begin{array}{l}\text { Autorrelato (FAPIS), exercício } \\
\text { de caso clínico (FAP-Vinhetas }{ }^{\text {a }} \text { ) } \\
\text { e observação com escala por } \\
\text { vídeo (FAPCS - sessão) }\end{array}$ & $\begin{array}{l}\text { Ensaio clínico randomizado/ GE }(n=8) \text { O X O O (follow up) } \\
\text { controle com lista de espera GC-LE }(n=8) \text { O O X O } \\
2 \text { meses }\end{array}$ \\
\hline \multirow[t]{2}{*}{$\begin{array}{l}\text { Almeida et al. } \\
(2016)\end{array}$} & Intimidade & $\begin{array}{l}\text { Avaliação teórica (própria) e } \\
\text { categorização de cenário de role } \\
\text { play por vídeo (FAPRS - teste) }\end{array}$ & Pré-experimento GE $(n=6) O X O$ \\
\hline & & & $\begin{array}{l}\text { Delineamentos de sujeito único (sessões) } \\
\text { Tipo Participante Sessões }\end{array}$ \\
\hline $\begin{array}{l}\text { Fonseca } \\
(2016)\end{array}$ & $\begin{array}{l}\text { Habilidades } \\
\text { FAP }\end{array}$ & $\begin{array}{l}\text { Autorrelato (FAPIS) e } \\
\text { categorização de comportamen- } \\
\text { to por comportamento por vídeo } \\
\text { (FAPRS - sessão) }\end{array}$ & 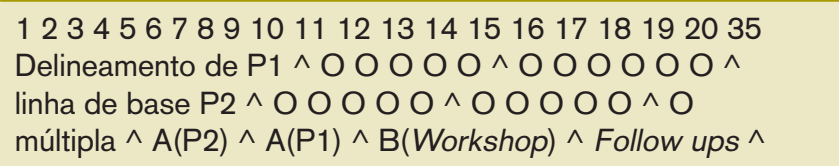 \\
\hline $\begin{array}{l}\text { Silveira et al. } \\
(2009)\end{array}$ & $\begin{array}{l}\text { Identificação } \\
\text { de CRBs }\end{array}$ & $\begin{array}{l}\text { Autorrelato (FIAT, FIAT-T e } \\
\text { PRCRB) e observação com } \\
\text { checklist em sala de espelho } \\
\text { (PRCRB - sessão) }\end{array}$ & $\begin{array}{l}1234567891011121314151617181920 \\
\text { Delineamento } \mathrm{P} 1 \mathrm{O} O \mathrm{O} O \mathrm{O} O \mathrm{O} O \mathrm{O} O \mathrm{O} O \mathrm{O} O \mathrm{O} \\
\mathrm{AB} \wedge \mathrm{A}(\mathrm{Sp} . \text { Comum }) \wedge \mathrm{B}>(\mathrm{Sp} \text {. FAP })\end{array}$ \\
\hline $\begin{array}{l}\text { Meurer } \\
(2011)\end{array}$ & $\begin{array}{l}\text { Habilidades } \\
\text { FAP }\end{array}$ & $\begin{array}{l}\text { Autorrelato (BDI e IHS) e cate- } \\
\text { gorização de comportamento } \\
\text { por vídeo (FAPRS - sessão) }\end{array}$ & $\begin{array}{l}1234567891011121314151617181920 \\
\text { Delineamento de P1 O O O O O O O O O O O O } \\
\text { reversão - ABA P2 O O O O O O O O O O O O } \\
\wedge A(\text { Sem } \mathrm{sp} .) \wedge \mathrm{B}>(\mathrm{Sp} \text {. FAP }) \wedge A(\text { Sem } \mathrm{sp} .)\end{array}$ \\
\hline $\begin{array}{l}\text { Lepienski e } \\
\text { Silveira(2017) }\end{array}$ & $\begin{array}{l}\text { Habilidades } \\
\text { FAP }\end{array}$ & $\begin{array}{l}\text { Categorização de comportamen- } \\
\text { to por vídeo (FAPRS - sessão e } \\
\text { supervisão) }\end{array}$ & $\begin{array}{l}1234567891011121314151617181920 \\
\text { Delineamento P1 O O O O O O O O O O O O O O O } \\
\mathrm{AB}^{\wedge} \mathrm{A}(\mathrm{Sp} \text {. Comum })^{\wedge} \mathrm{B}>(\mathrm{Sp} \text {. FAP) }\end{array}$ \\
\hline
\end{tabular}

Nota. FAPIS = FAP Impact Scale (Kanter et al., 2013); IRI = Interpersonal Reactivity Index (Davis, 1983); CLS = Compassionate Love Scale: Stranger-humanity Version (Sprecher \& Fehr, 2005); FFMQ-SF = Five Facet Mindfulness Questionnaire-Short Form (Bohlmeijer, ten Klooster, Fledderus, Veehof, \& Baer, 2011); AS = Authenticity Scale (Wood, Linley, Maltby, Baliousis, \& Joseph, 2008); TEI = Treatment Evaluation Inventory (Kazdin, 1980); MC-SDS = Marlowe-Crowne Social Desirability Scale-Short Form C (Reynolds, 1982); FAPCS = FAP Competency Scale (instrumento não publicado e desenvolvido pelo segundo autor do estudo de Maitland et al., 2016, no qual é apresentada uma breve descrição); FAPRS = FAP Rating Scale (Callaghan, Follette, Ruckstuhl, \& Linnerooth, 2008); PRCRB = Protocolo de Registro de Comportamentos Clinicamente Relevantes (Silveira et al., 2009); BDI = Beck Depression Inventory (Beck, Ward, Mendelson, Mock, \& Erbaugh, 1961); IHS = Inventário de Habilidades Sociais (Del Prette, \& Del Prette, 2001). Foram utilizadas algumas notações científicas para descrever os delineamentos utilizados. $\mathrm{GE}=$ grupo experimental; $\mathrm{GC}=$ grupo controle; $\mathrm{LE}=$ lista de espera; $\mathrm{O}=$ aplicação do teste; $\mathrm{X}=$ aplicação da intervenção; $\mathrm{A}=$ linha de base; $\mathrm{B}=$ intervenção; ${ }^{\wedge}=$ indica o momento da introdução da linha de base ou da intervenção; Sp. = supervisão. anstrumento desenvolvido e descrito no estudo de Kanter et al. (2013). 
Como pode-se observar na Tabela 3 , dos oito estudos experimentais, metade utilizou delineamentos entre grupos, e a outra metade optou por delineamentos de sujeito único.

Estudos com delineamento entre grupos. Três dos quatro delineamentos entre grupos empregaram ensaios clínicos randomizados com a condição controle de lista de espera, que significa que esse grupo será submetido a intervenção posteriormente ao grupo experimental. Um desses estudos utilizou um delineamento padrão: teste 1 - treino - teste 2 no grupo experimental, enquanto o grupo controle fez os testes, mas não recebeu o treino durante o andamento do estudo. Nos outros dois estudos o grupo controle recebeu o treino após o teste 2 do grupo experimental, gerando um delineamento teste 1 - treino - teste 2 no grupo experimental e teste 1 - teste 2 - treino - teste 3 no grupo controle (os testes 1 e 2 foram aplicados ao mesmo tempo nos dois grupos). Dois desses estudos também contaram com um pequeno follow up, dois meses após a intervenção.

O outro dos quatro delineamentos entre grupos foi um estudo pré-experimental, sem grupo controle, que investigou um dos dois workshops. Esse tipo de delineamento tem limitações importantes no controle experimental, que geram limitações nas conclusões deste tipo de estudo (Bootzin \& Ruggill, 1988). As amostras por condição são consideradas pequenas (Kanter et al., 2017), variando entre 6 e 13, o que pode diminuir o poder das inferências estatísticas (Hill \& Lent, 2007).

A variável dependente (VD) habilidades FAP (relacionadas as 5 regras da FAP, que envolvem identificar, evocar e reforçar CRBs) foi a mesma nos três estudos de THT. As habilidades FAP nesses estudos foram medidas com instrumentos de autorrelato e exercícios de caso clínico. Um desses estudos (Maitland et al., 2016) também utilizou um método de observação de gravações em vídeos por registro em escalas. O estudo de Keng et al. (2016) também incluiu avaliações, por meio de instrumentos de autorrelatos, de constructos como: empatia, amor, mindfulness-traço e autenticidade. $\mathrm{O}$ estudo sobre workshop de Almeida et al. (2016) avaliou apenas a intimidade (VD) por meio de uma avaliação teórica e da categorização por observadores de comportamentos em cenário de role play gravado em vídeo.

Tomados como um todo, os estudos que utilizaram delineamentos entre grupos priorizaram instrumentos de autorrelato, utilizados em três estudos. Beidas e Kendall (2010) chamaram a atenção para o fato de que reportar a mudança de comportamento não implica necessariamente na mudança de comportamento. Para os autores, acreditar na relação entre o relato e o comportamento em si é problemático e essa crença poderia levar a conclusões equivocadas sobre a eficácia dos treinamentos sobre a aquisição de habilidades terapêuticas. Em vez disso, Beidas e Kendall, assim como Hill e Lent (2006), sugerem a utilização de medidas diretas de comportamentos como uma alternativa mais confiável. Essa alternativa foi utilizada pelos estudos revisados ao observar comportamentos por vídeos ou ao vivo e categorizar por meio de um checklist ou sistema de categorização e é defendida por Weeks et al. (2011) para estudos de ensino de FAP.

Os três estudos de THT investigaram o mesmo protocolo de ensino e são vinculados ao que parece ser um mesmo grupo de pesquisa localizado em Seattle. Esse grupo parece priorizar a investigação de THTs por meio de delineamentos entre grupos, em contrariedade as críticas de analistas do comportamento a essa proposta (Skinner, 1956; Sidman, 1960/1976). Esses estudos têm produzido um bom corpo de evidências em relação ao mesmo protocolo. Em relação à pesquisa original de Kanter, os estudos de Keng et al. (2016) e Maitland et al. (2016) acrescentaram períodos de follow up, instrumentos de análise por categorização por vídeos, verificaram o efeito do treino sobre outras habilidades, incluíram nas estratégias de ensino leituras didáticas, testaram o mesmo treinamento em uma modalidade presencial e testaram o protocolo em uma cultura diferente, além de aumentar o número de participantes submetidos ao mesmo protocolo, o que permitirá uma avaliação futura por meta-análise.

Estudos com delineamentos de sujeito único. Em relação aos quatro estudos que utilizaram delineamento de sujeito único, todos foram vinculados a instituições brasileiras (sendo uma colaboração internacional), indicando uma predileção des- 
ses pesquisadores por esse tipo de metodologia experimental, ao contrário da preferência observada nas pesquisas de instituições americanas. Delineamentos de sujeito únicos tem sido estratégias metodológicas preferidas para pesquisas em análise do comportamento (Johnston \& Pennypacker. 1993; Sidman, 1960/1976) e as mais utilizadas para pesquisas de eficácia em FAP (Kanter et al., 2017). Desses quatro estudos que utilizaram delineamentos de sujeito único, três deles avaliaram a estratégia de ensino de supervisão e outro a estratégia de workshop. O que pode indicar uma relação entre o método de ensino e o delineamento empregado. Todos os estudos experimentais de supervisão utilizaram delineamento de sujeito único, o formato normalmente individualizado dessa estratégia de treinamento pode favorecer essa opção. No mesmo sentido, todos os estudos de THT, que foram em grupo, optaram por utilizar delineamentos entre grupos. No caso dos dois workshops, que também contam com um formato de treinamento em grupo, um utilizou um delineamento entre grupos e o outro, o estudo de Fonseca (2016), optou por acompanhar as sessões de atendimento de um participante antes e após a participação deste em um workshop em grupo, demonstrando uma possibilidade de utilização de um delineamento de sujeito único em uma estratégia de formato em grupo.

O tipo de delineamento de sujeito único mais utilizado foi o A-B (linha de base e intervenção), com duas ocorrências. Os outros delineamentos utilizados foram: um delineamento de reversão A-B-A - linha de base, intervenção e linha de base novamente - replicado em um segundo sujeito; e um delineamento de linha de base múltipla, no qual o tratamento de um segundo sujeito iniciou-se algumas semanas depois, e com follow up. Réplicas intra (reversão) e entre sujeitos (aplicação a novos sujeitos) aumentam, respectivamente, a validade interna e externa do delineamento empregado (Sidman, 1960/1976). Em três dos quatro estudos de delineamento de sujeito único, houve cinco ou mais medições de comportamento por condição, cuidado metodológico importante para delineamentos de sujeito único de alta qualidade (Smith, 2012). A exceção foi o estudo de Meurer (2011), que atingiu o critério de cinco medições durante a linha de base, porém contou com apenas 4 medições durante a intervenção e 3 medições após a retirada da intervenção.

Em relação as variáveis dependentes dos estudos de delineamentos de sujeito único, em três estudos foram as habilidades FAP e no outro o objetivo do treinamento foi ensinar o terapeuta a identificar CRBs (VD), um dos componentes das habilidades FAP. Essas VDs foram mensuradas por meio de instrumentos de autorrelatos (três estudos) e categorização de comportamentos observados por vídeo (3 estudos) e observação direta por sala de espelho com uso de checklist (1 estudo). Em geral, a maioria dos estudos experimentais, independentemente do tipo de delineamento utilizado, focou no ensino de habilidades típicas da FAP e mediram essas habilidades por meio de instrumentos de autorrelato (6 estudos) e de procedimentos de observação e registro de comportamentos (6 estudos), por vídeo (5 estudos) ou por sala de espelho ( 1 estudo) estudo, na sessão (5 estudos) ou em cenário de role play (5 estudos).

Estudos de caso. Dois dos manuscritos encontrados (Sousa \& Vandenberghe, 2007; Wielenska \& Oshiro, 2012) foram classificados como estudos de caso. Ambos descreveram trechos de supervisões com uma análise qualitativa e não apresentavam uma comparação clara dos resultados antes e depois da supervisão. O estudo de Sousa e Vandenberghe (2007), o mais antigo encontrado na revisão, é um estudo de caso que analisa as possibilidades de treino de terapeutas FAP por supervisão, ao explorar as similaridades funcionais entre o processo de terapia e de supervisão. A supervisão foi realizada por uma psicóloga, em 2 ou 3 sessões mensais individuais, para 4 terapeutas, com experiência clínica prévia, que estavam trabalhando com pacientes diagnosticados com o Transtorno de Personalidade Borderline. Os casos foram analisados por meio da descrição de trechos da supervisão que exemplificavam duas dificuldades comuns entre esses terapeutas, que foram categorizadas como esquiva experiencial e desespero.

O artigo de Wielenska e Oshiro (2012) apresentou a descrição de dois estudos de caso. Ambos descreveram o funcionamento de um programa de ensino de FAP, na USP, com a diferença de que o primeiro estudo teve como população alvo estu- 
dantes de pós-graduação em psicologia e o segundo foi realizado com residentes de psiquiatria. Apenas o primeiro estudo de caso foi incluído. O segundo estudo de caso, ao ser direcionado ao treinamento de estudantes de psiquiatria, não atendeu o critério de inclusão deste estudo, de ser um treinamento para psicólogos graduandos ou formados. O estudo de caso descreveu trechos de uma supervisão FAP em grupo - de duas horas semanais, com uma supervisora e 14 terapeutas de um programa de formação para terapeutas formados com diferentes backgrounds - para ilustrar esse tipo de intervenção e fornecer subsídios para futuros estudos. Os supervisionandos também recebiam concomitantemente quatro horas de aulas teóricas semanais e atendiam em duplas pacientes de um hospital universitário com diferentes problemas.

Nos dois estudos de caso não foram descritos os procedimentos de intervenção, embora nas descrições das intervenções possam ser observados o uso, pelas supervisoras, de procedimentos semelhantes aos utilizados em uma terapia FAP - que seriam os mesmos procedimentos que os terapeutas deveriam aprender, ao ter a supervisão como modelo - como evocação de CRB1, modelagem de CRB2 e CRB3, além do uso de discussão dos casos com feedback e instruções e bloqueio de esquiva. Os dois manuscritos relataram que esses procedimentos foram importantes para o desenvolvimento dos terapeutas e para a condução dos casos clínicos.

Diferente dos delineamentos experimentais, os estudos de caso implicam em limitações quanto: (a) à validade interna, pois causalidade não pode ser atribuída as relações observadas no estudo de caso, por não incluir manipulação e controle experimental; e (b) à validade externa, uma vez que os resultados só foram observados naquele grupo e em uma determinada situação, eles não podem ser generalizados para outras condições e outros sujeitos (Cozby, 2003). Além disso, nesses dois estudos, algumas informações não foram adequadamente descritas - o que dificulta a replicabilidade e a confiança nos resultados: (a) a experiência dos treinadores, (b) os procedimentos de intervenção e (c) os instrumentos e medidas utilizadas.

Estudos correlacionais. Os estudos de Knott et al. (2015) e Terry e Kohlenberg (2012) utilizaram métodos correlacionais, no qual não há manipulação experimental. Nos dois estudos citados uma população de psicoterapeutas foi dividida em dois grupos, os que alegavam ter tido uma formação em FAP e os terapeutas formados em outras orientações teóricas, ou seja, a variável independente - formação em FAP - não foi manipulada, os participantes não foram submetidos a um mesmo treino em FAP. Para serem considerados terapeutas formados em FAP os participantes deveriam ter ao menos uma de duas experiências, treino formal ou prática em FAP. O tempo da treino e de experiencia prática em FAP variou bastante entre os participantes, e o tipo de treino em FAP não foi categorizado nos estudos, apenas foi relatado o tempo das formações (que variou bastante), o que sugere que os tipos de formação possam ter sido muito diferentes entre os participantes. Os estudos correlacionaram a variável independente (formação em FAP ou formação não-FAP) com uma variável dependente, alguma característica comportamental do terapeuta mensurada, que foi diferente para cada estudo.

Knott, et al. (2015) avaliaram o efeito da formação em FAP sobre as habilidades de intimidade e autorrevelação de 80 terapeutas (26 treinados em FAP). Para isso os terapeutas responderam a dois instrumentos de avaliação, o Functional Analytic Psychotherapy Intimacy Scale (FAPIS; Leonard et al., 2014) e o Functional idiographic assessment template: disclosure subclass (FIAT-QD; Callaghan, 2006b, vale ressaltar que autorrevelação é uma variável medida justamente pela classe D do FIAT). Observou-se que os terapeutas que possuíam formação em FAP obtiveram um escore maior na escala de intimidade, FAPIS, no contexto terapêutico, do que terapeutas que foram treinados em outras propostas terapêuticas. Não houve diferenças significativas em relação a medida de autorrevelação, FIAT-QD.

Terry e Kohlenberg (2012) aplicaram em 193 psicólogos (49 treinados em FAP) o Therapeutic Relationship Measure (TRM), um instrumento desenvolvido pelos autores, e descrito no próprio estudo, com escalas tipo Likert, e uma adaptação do teste de associação implícita (Implicit Association Test - IAT; Greenwald, McGhee, \& Schwartz, 1998) que mediam, respectivamente, atitudes explícitas e implícitas em relação ao uso de intervenções psicoterápicas focadas na relação terapêutica. Os tera- 
peutas que possuíam formação em FAP mostraram atitudes explícitas e implícitas mais positivas em relação ao uso de intervenções focadas na relação quando comparados a terapeutas que não foram formados em FAP; esses dados são condizentes com a revisão de Beidas e Kendall (2010), na qual se observou que treinamentos em psicoterapias são efetivos para mudar atitudes.

A validade das conclusões desses estudos é limitada devido a metodologia empregada. Segundo Cozby (2003), os estudos correlacionais não empregam uma manipulação experimental e não controlam possíveis variáveis que possam estar intervindo na relação observada, ao invés disso esses estudos relacionam a variação conjunta de determinadas características de uma população. Esse tipo de procedimento de pesquisa não consegue atribuir causalidade às relações observadas, embora seja importante para o levantamento de possíveis relações entre variáveis que depois possam ser testadas experimentalmente.

Além desse problema intrínseco aos estudos correlacionais, outros problemas metodológicos foram observados. Os participantes dos dois estudos passaram por formações em FAP muito diversas, podendo ser um treino formal ou mesmo a prática sem treino formal. O tempo de treino e prática variou bastante entre os participantes, e aqueles que passaram por um treino formal, não tiveram o tipo de treino especificado. A diversidade do tipo de formação em FAP impossibilita um exame da relação entre o tipo específico de formação e a variação média do repertório comportamental medido na população. Outro problema é que a definição de atitude de Terry e Kolenberg (2012) não é adequadamente operacionalizada. Atitudes são constructos, derivados da psicologia social, normalmente interpretados como cognições que mediam as relações entre estímulo e resposta (embora uma interpretação analítico-comportamental já tenha sido proposta, ver Guerin, 1994). Terry e Kohlenberg (2012) definiram atitudes ou preferências como aproximação ou afastamento das oportunidades de intervenções focadas na relação; essa definição não especifica as relações que o responder do indivíduo estabelece com o ambiente, o que significa, em uma visão behaviorista radical, que esse constructo não está sendo tratado enquanto comportamento.
Os instrumentos de medida utilizados nesses dois estudos também possuem limitações importantes: (a) com exceção do IAT, todos os outros instrumentos utilizaram medidas de autorrelato, que mensuram o falar sobre o comportamento e não o comportamento em si; (b) nos instrumentos FAPIS e TRM essas medidas de autorrelato descreveram a topografia de respostas privadas e públicas, sem praticamente nenhuma relação com variáveis ambientais externas; e (c) com exceção do FIAT-QD, os outros instrumentos mediram constructos hipotéticos; embora no trabalho de Knott et al. (2015) a intimidade seja definida operacionalmente, na FAPIS ela é tratada como um constructo definido pela sua topografia; enquanto que no IAT o constructo preferência é inferido pela latência da resposta. Todas essas limitações dos instrumentos utilizados são incompatíveis com uma proposta terapêutica que se sustenta na análise do comportamento, que defende medidas de observação direta do comportamento, avaliação da relação entre resposta e ambiente e a não utilização de constructos hipotéticos (ver Johnston \& Pennypacker, 1993).

\section{Considerações Finais}

Esta revisão expande o número de manuscritos encontrados pela revisão anterior de Kanter et al. (2017), os autores haviam encontrado apenas 3 artigos (Kanter, et al., 2013; Keng, et al., 2016; Maitland, et al., 2016). Outros 2 artigos incluídos no presente estudo foram citados (Silveira, et al., 2009; Sousa \& Vandenberghe, 2007), embora não tenham sido incluídos pelos critérios do estudo de Kanter et al. (2017). Essa diferença provavelmente ocorreu por alguns motivos: (a) a revisão não englobou apenas estudos de treinamento terapêutico; (b) não foram utilizados bancos de dados que incluam periódicos importantes de literatura brasileira, embora Kanter et al. (2017) tenham incluso trabalhos em português; (c) incluíram apenas trabalhos com análise quantitativa. Considerando que as pesquisas sobre essa temática são recentes, a presente revisão teve um cunho mais exploratório, por essa razão flexibilizou na metodologia os critérios de inclusão dos estudos, o que possibilitou um levantamento de toda a produção e uma análise crítica das publicações. 
Por meio dessa análise, observou-se alguns aspectos que podem ser aperfeiçoados como diretrizes para estudos experimentais de alta qualidade, segundo as observações de Hill e Lent (2006): (a) amostra maior nos delineamentos entre grupos; (b) maior número de replicações intra e entre sujeitos nos delineamentos de sujeito único (Smith, 2012); (c) maiores períodos de follow up; (d); utilização de protocolos nas supervisões clínicas (e); menos participantes por treino; (f) treinos de maior duração.

Os estudos de caso, devido as suas limitações intrínsecas quanto a validade interna e externa, deveriam ser utilizados apenas em estágios iniciais da pesquisa, em que ainda não há literatura experimental ou correlacional sobre o tema, como é o caso dos dois estudos de caso aqui analisados, Silva e Vandenberghe (2007) e Wielenska e Oshiro (2012); ou quando fosse observada durante a aplicação de um procedimento de treino alguma "condição particular, incomum ou notável” (Cozby, 2004, p.134), que seria útil para levantar novas hipóteses, que serviriam para novos estudos experimentais. Em outros casos, os estudos de caso deveriam ser desincentivados, e deveriam ser preferíveis estudos correlacionais e, principalmente, experimentais.

Em relação aos estudos correlacionais, alguns aspectos que poderiam aumentar a sua qualidade metodológica: (a) definir operacionalmente as variáveis medidas, evitando construtos hipotéticos; (b) adicionar medidas alternativas aos autorrelatos, incluindo medidas observacionais; e (c) investigar uma amostra composta por sujeitos que foram submetidos a um mesmo tipo de treinamento em FAP.

Além disso, futuros estudos poderiam: (a) promover maiores intercâmbios entre os estudiosos de diferentes grupos de pesquisa; (b) investigar outras estratégias de ensino, como manuais e o uso de softwares para o ensino autoguiado; (c) investigar programas que incluam diversas estratégias de ensino, como formações e pós-graduações em FAP. Essas diretrizes podem aumentar a produção e a qualidade dos treinamentos em FAP, que por sua vez, permitirão uma adequada implementação e disseminação da FAP. 


\section{Referências}

Alfonsson, S., Parling, T., Spannargard, A., Andersson, G., \& Lundgren, T. (2017). The effects of clinical supervision on supervisees and patients in cognitive behavioral therapy: A systematic review. Cognitive-Behavior Therapy, 47(3), 206-228. https://doi.org/10.1080/165060 73.2017.1369559

Almeida, M. S., Runnacles, A. L. E. S., \& Silveira, J. M. (2016). Treino de comportamentos de intimidade para terapeutas em processo de formação em Psicoterapia Analítica Funcional. Perspectivas em Análise do Comportamento, 7, 212-228. http://dx.doi.org/10.18761/ pac. 2016.020

Beck, A. T., Ward, C. H., Mendelson, M., Mock, J., \& Erbaugh, J. (1961). An inventory for measuring depression. Archives of General Psychiatry, 4, 561-571. https://doi.org/10.1001/archpsyc.1961.01710120031004

Beidas, R. S., \& Kendall, P. C. (2010). Training therapists in evidence-based practice A critical review of studies from a systems-contextual perspective. Clinical Psychology: Science and Practice, 17(1), 1-30. https://doi.org/10.1111/ j.1468-2850.2009.01187.x

Beidas, R. S., Koerner, K., Weingardt, K. R., \& Kendall, P. C. (2011). Training research: practical recommendations for maximum impact. Administration and Policy in Mental Health and Mental Health Services Research, 38, 223-237. https://doi.org/10.1007/s10488-011-0338-z

Berger, T. (2004). Computer-based technological applications in psychotherapy training. Journal of Clinical Psychology, 60(3), 301-315. doi:10.1002/jclp.10265

Bohlmeijer, E., ten Klooster, P. M., Fledderus, M., Veehof, M., \& Baer, R. (2011). Psychometric properties of the Five Facet Mindfulness Questionnaire in depressed adults and development of a short form. Assessment, 18, 308-320. https://doi.org/10.1177/1073191111408231

Bootzin, R. R., \& Ruggill, J. S. (1988). Training issues in behavior therapy. Journal of Consulting and Clinical Psychology, 56(5), 703-770. http:// dx.doi.org/10.1037/0022-006X.56.5.703
Callaghan, G. M. (2006a). Functional analytic psychotherapy and supervision. International Journal of Behavioral Consultation and Therapy, 2(3), 416-431. http://dx.doi.org/10.1037/ h0100794

Callaghan, G. M. (2006b). The Functional Idiographic Assessment Template (FIAT) System: For use with interpersonally-based interventions including Functional Analytic Psychotherapy (FAP) and FAP-enhanced treatments. The Behavior Analyst Today, 7, 357-398. http://dx.doi.org/10.1037/h0100160

Callaghan, G. M., Follette,W. C., Ruckstuhl, L. E., Jr., \& Linnerooth, P. J. (2008). The Functional Analytic Psychotherapy Rating Scale (FAPRS): A behavioral psychotherapy coding system. The Behavior Analyst Today, 9(1), 98-116. http:// dx.doi.org/10.1037/h0100648

Cozby, P. C. (2003). Métodos de pesquisa em ciência do comportamento. São Paulo: Atlas.

Davis, M. H. (1983). Measuring individual differences in empathy: Evidence for a multidimensional approach. Journal of Personality and Social Psychology, 44, 113-126. https://doi. org/10.1037/0022-3514.44.1.113

Decker, S. E., Jameson, M. T., \& Naugle, A. E. (2011). Therapist training in empirically supported treatments: A review of evaluation methods for short- and long-term outcomes. Administration and Policy in Mental Health and Mental Health Services Research, 38(4), 254-286. https://doi. org/10.1007/s10488-011-0360-1

Del Prette, Z. A. P., \& Del Prette, A. (2001). Inventário de Habilidades Sociais: Manual de aplicação, apuração e interpretação. São Paulo: Casa do Psicólogo.

Follette, W. C., \& Callaghan, G. M. (1995). Do as I do, not as I say: A behavior-analytic approach to supervision. Professional Psychology: Research \& Practice, 26(4), 413-421. http:// dx.doi.org/10.1037/0735-7028.26.4.413

Fonseca, N. M. (2016). Efeitos de workshop de psicoterapia analítica-funcional sobre habilidades terapêuticas (Dissertação de Mestrado). Universidade de São Paulo, São Paulo.

Greenhalgh, T., Robert, G., MacFarlane, F., Bate, P., \& Kyriakidou, O. (2004). Diffusion of innovations in service organizations: Systematic re- 
view and recommendations. Milbank Quarterly, 82(4), 581-629. https://doi.org/10.1111/j.0887378X.2004.00325.x

Greenwald, A. G., McGhee, D. E., \& Schwartz, J. L. K. (1998). Measuring individual differences in implicit cognition: The Implicit Association Test. Journal of Personality and Social Psychology, 74(6), 1464 - 1480.

Guerin, B. (1994). Analyzing Social Behavior: Behavior analysis and the social sciences. Reno, NV: Context Press.

Herschell, A. D., Kolko, D. J., Baumann, B. L., \& Davis, A. C. (2010). The role of therapist training in the implementation of psychosocial treatments: A review and critique with recommendations. Clinical Psychology Review, 30(4), 448-466. https://doi.org/10.1016/j. cpr.2010.02.005

Hill, C. E., \& Lent, R. W. (2006). A narrative and meta-analytic review of helping skills training: time to revive a dormant area of inquiry. Psychotherapy: Theory, Research, Practice and Training. 43(2), 154-172. http://dx.doi. org/10.1037/0033-3204.43.2.154

Holman, G., Kohlenberg, R. J., \& Tsai, M. (2012). Development and preliminary evaluation of a FAP protocol: Brief relationship enhancement. International Journal of Behavioral Consultation and Therapy, 7(2-3), 52-57. http://dx.doi. org/10.1037/h0100937

Johnston, J. M., \& Pennypacker, H. S. (1993). Readings for strategies and tactics of behavioral research (2a ed.). Hillsdale, NJ: Lawrence Erlbaum.

Kanter, J. W., Manbeck, K. E., Kuczynski, A. M., Maitland, D. W. M., Villas-Boas, A., \& Ortega, M. A. R. (2017). A comprehensive review of research on Functional Analytic Psychotherapy. Clinical Psychology Review, 58, 141-156. http:// dx.doi.org/10.1016/j.cpr.2017.09.010

Kanter, J. W., Tsai, M., Holman, G., \& Koerner, K. (2013). Preliminary data from a randomized pilot study of web-based functional analytic psychotherapy therapist training. Psychotherapy, 50(2), 248-255. http://dx.doi.org/10.1037/ a0029814

Kazdin, A. E. (1980). Acceptability of alternative treatments for deviant child behavior. Journal of
Applied Behavior Analysis, 13, 259-273. https:// doi.org/10.1901/jaba.1980.13-259

Keng, S. L., Waddington, E., Lin, X. B., Tan, M. S. Q., Henn-Haase, C., \& Kanter, J. K. (2016). Effects of functional analytic psychotherapy therapist training on therapist factors among therapist trainees in Singapore: A randomized controlled trial. Clinical Psychology and Psychotherapy, 24(4), 1014-1027. https://doi. org/10.1002/cpp.2064

Knott, L. E., Wettemeck, C. T., Derr, D., \& Tolentino, R. (2015). A functional analytic perspective of therapist intimacy in and out of session. International Journal of Behavioral Consultation and Therapy, 9(4), 6-10.

Kohlenberg, R. J., \& Tsai, M. (1987). Functional analytic psychotherapy. Em N. S. Jacobson (Ed.), Psychotherapists in clinical practice: Cognitive and behavioral perspectives (pp. 388-443). New York: Guilford.

Kohlenberg, R. J., \& Tsai, M. (1991). Functional analytic psychotherapy: Creating intense and curative therapeutic relationships. New York: Plenum Press.

Kohlenberg, R. J., Hayes, S. C., \& Tsai, M. (1993). Radical behavioral psychotherapy: Two comtemporary examples. Clinical Psychology Review, 13, 579-592. https://doi. org/10.1016/0272-7358(93)90047-P

Leonard, R. C., Knott, L. E., Lee, E. B., Singh, S., Smith, A. H., Kanter, J., (...), \& Wetterneck, C. T. (2014). The development of the functional analytic psychotherapy intimacy scale. The Psychological Record, 64(4), 647-657. https:// doi.org/10.1007/s40732-014-0089-9

Lepienski, A. F. \& Silveira, J. M. (2017). Relações entre uma supervisão em psicoterapia analítica funcional e as respostas de uma terapeuta e sua cliente em sessão. Acta Comportamentalia, 25(3), 347-363. Retirado de: http:// www;redalyc.org/articulo.oa?id=274552568004 Maitland, D. W. M., Kanter, J. W., Tsai, M. Kuczynski, A. M., Manbeck, K. E., \& Kohlenberg, R. J. (2016). Preliminary findings on the effects of online Functional Analytic Psychotherapy training on therapist competence. Psychological Record, 66(4), 627-637. https://doi.org/10.1007/ s40732-016-0198-8 
Mangabeira, V., Kanter, J., \& Del Prette, G. (2012). Functional Analytic Psychotherapy (FAP): A review of publications from 1990 to 2010. International Journal of Behavioral Consultation and Therapy, 7(2-3), 78-89. http://dx.doi. org/10.1037/h0100941

Meurer, P. H. (2011). Efeito da apresentação de feedback no comportamento do terapeuta de evocar e responder aos comportamentos clinicamente relevantes (Dissertação de Mestrado). Universidade Federal do Paraná, Curitiba.

Miller, S. J., \& Binder, J. L. (2002). The effects of manual-based training on treatment fidelity and outcome: A review of the literature on adult individual psychotherapy. Psychotherapy: Therapy/Research/Practice/Training, 39(2), 184-198. http://dx.doi.org/10.1037/00333204.39.2.184

Paiva, M. R. F., Parente, J. R. F., Brandão, I.R ., \& Queiroz, A. H. B. (2016). Metodologias ativas de ensino-aprendizagem: revisão integrativa. Sanaré, 15(2), 145-153.

Prince, M. (2004). Does active learning work? A review of the research. Journal of Engineering Education, 93(3), 223-231. https://doi. org/10.1002/j.2168-9830.2004.tb00809.x

Reynolds, W. M. (1982). Development of reliable and valid short forms of the Marlowe-Crowne Social Desirability Scale. Journal of Clinical Psychology, 38, 119-125. https://doi.org/10.1002/10974679 ( 198201$) 38: 1<119$ : : A I D JCLP2270380118>3.0.CO;2-I

Rousmaniere, T. (2014). Using technology to enhance clinical supervision and training. Em C. E. Warkins, Jr. \& D. L. Milne (Orgs.). The Wiley International Handbook of Clinical Supervision ( $1^{\text {a }}$ ed.). West Sussex, UK: John Wiley \& Sons, Ltd.

Sidman, M. (1976). Táticas da pesquisa científica: Avaliação dos dados experimentais na psicologia (M. E. Paiva, Trad.). São Paulo, SP: Brasiliense. (Original publicado em 1960)

Silveira, J. M., Callaghan, G. M., Stradioto, A., Maeoka, B. E., Maurício, M. N., \& Goulin, P. (2009). Efeitos de um treino em psicoterapia analítica funcional sobre a identificação feita pelo terapeuta de comportamentos clinicamente relevantes de seu cliente. Revista Brasileira de
Terapia Comportamental e Cognitiva, 11(2), 346-365. https://doi.org/10.31505/rbtcc. v11i2.409

Skinner, B. F. (1956). A case history in scientific method. The American Psychologist, 11, 221233. http://dx.doi.org/10.1037/h0047662

Skinner, B. F. (1968) The technology of teaching. Acton: Copley Publishing Group.

Smith, J. D. (2012). Single-case experimental designs: A systematic review of published research and current standards. Psychological Methods, 17(4), 1-70. http://dx.doi.org/10.1037/a0029312

Sprecher, S., \& Fehr, B. (2005). Compassionate love for close others and humanity. Journal of Social and Personal Relationships, 22, 629-651. https:// doi.org/10.1177/0265407505056439

Sousa, A. C. A., \& Vandenberghe, L. (2007). Possibilidades da FAP como método de supervisão de terapeutas com clientes Boderlines. Revista Brasileira de Terapia Cognitiva e Comportamental, 9(1), 1-11. https://doi. org/10.31505/rbtcc.v9i1.142

Southam-Geraw, M. A., \& McLeod, B. D. (2013). Advances in applying treatment integrity research for dissemination and implementation science. Clinical Psychology Science and Practice, 20, 1-13. https://doi.org/10.1111/cpsp.12019

Terry, C. M., \& Kohlenberg, R. J. (2012). Therapists' attitudes about and preferences to use relationship focused interventions: New tools to measure a critical component of functional analytic psychotherapy (FAP). International Journal of Behavioral Consultation and Therapy, 7(2-3), 138-146. http://dx.doi.org/10.1037/h0100949

Tsai, M., Callaghan, G. M., Kohlenberg, B., Follette, W. C., Darrow, S. M. (2009). Supervision and therapist self-development. Em M. Tsai, R. J. Kohlenberg, J. W. Kanter, B. Kohlenberg, W. C. Follete \& G. M. Callaghan (Orgs.). A guide to Functional Analytic Psychotherapy: Awareness, courage, love and behaviorism (p. 167-198) New York: Spring.

Tsai, M., Kohlenberg, R. J., Kanter, J. W., Kohlenberg, B., Follete, W. C. \& Callaghan, G. M. (2009). A guide to Functional Analytic Psychotherapy: Awareness, courage, love and behaviorism. New York: Spring.

Tsai, M., Yard, S., \& Kohlenberg, R. J. (2014). 
Functional analytic psychotherapy: A behavioral relational approach to treatment. Psychotherapy, 51(3), 364-371. http://dx.doi. org/10.1037/a0036506

Vandenberghe, L. (2009a). Keeping the focus on clinically relevant behavior: Supervision for Functional Analytic Psychotherapy. International Journal of Behavioral Consultation and Therapy, 5(2), 209-221.

Vandenberghe, L. (2009b). A functional analytic approach to group psychotherapy. The Behavior Analyst Today, 10(1), 71-82. http://dx.doi. org/10.1037/h0100665

Vandenberghe, L. (2011). Terceira onda e terapia analítico-comportamental: Um casamento acertado ou companheiros de cama estranhos? Boletim Contexto ABPMC, 34, 33-41.

Vandenberghe, L. (2017). Três faces da psicoterapia analítica funcional: uma ponte entre análise do comportamento e terceira onda. Revista Brasileira de Terapia Comportamental e Cognitiva, 19(3), 206-219. https://doi. org/10.31505/rbtcc.v19i3.1063

Walters, S. T., Matson, S. A., Baer, J. S., \& Ziedonis, D. M. (2005). Effectiveness of workshop training for psychosocial addiction treatment: A systematic review. Journal of Substance Abuse Treatment, 29, 283-293. https://doi. org/10.1016/j.jsat.2005.08.006

Wheeler, S., \& Richards, K. (2007). The impact of clinical supervision on counsellors and therapists, their practice and their clients. A systematic review of the literature. Counselling and Psychotherapy Research, 7, 54-65. https://doi. org/10.1080/14733140601185274

Wielenska, R. C., \& Oshiro, C. K. B. (2012). FAP group supervision: Reporting educational experiences at the University of São Paulo, Brazil. International Journal of Behavioral Consultation and Therapy, 7(2-3), 177-181. http://dx.doi. org/10.1037/h0100953

Wood, A. M., Linley, P. A., Maltby, J., Baliousis, M., \& Joseph, S. (2008). The authentic personality: A theoretical and empirical conceptualization and the development of the Authenticity Scale. Journal of Counseling Psychology, 55, 385-399. https://doi.org/10.1037/0022-0167.55.3.385

\section{Informações do Artigo}

Histórico do artigo:

Submetido em: 24/08/2019

Primeira decisão editorial: 30/10/2020

Aceito em: 03/11/2020

Editor associado: Daniel Afonso Assaz 